\title{
Ab-initio Coulomb Scattering in Atomistic Device Simulation
}

\author{
C. R. AROKIANATHAN, J. H. DAVIES and A. ASENOV* \\ Device Modelling Group, Department of Electronics and Electrical Engineering, \\ University of Glasgow, Glasgow G12 8QQ, Scotland, UK
}

\begin{abstract}
As devices shrink to dimensions below $0.1 \mu \mathrm{m}$, it becomes essential to treat impurities and carriers as individual charges. We describe some approaches to ionised impurity scattering where the potential of the impurities is included directly in the dynamics of a Monto Carlo type simulation rather than as a scattering rate. The divergence in the Coulomb potential creates difficulties for mesh-based solutions of Poisson's equation, which we have compared with more accurate Ewald summation. However, we find that the mesh does not introduce significant errors, and reproduces well the expected mobility as a function of doping. Highly accurate integration of the equation of motion is needed for free carriers, and the initial distribution is problematic. In contrast, a simple treatment of phonon scattering by Brownian dynamics is more tolerant of errors because it tends to restore the system to equilibrium.
\end{abstract}

Keywords: Semiconductors, discrete charges, numerical simulation, Monte Carlo, mobility

\section{INTRODUCTION}

As semiconductor devices shrink to dimensions below $0.1 \mu \mathrm{m}$, their properties begin to differ from larger structures as the atomistic nature of the charges begins to exert its influence [1]. The simulation of such devices requires a full-scale 3D treatment, including both impurities and carriers as individual particles [2,3]. This presents a severe computational load, both in the solution of Poission's equation and because the equation of motion must be integrated through a complex potential landscape. Rapid variation of the potential in space may create difficulties with meshbased calculations, both for the dynamics and Poisson's equation.

In this paper we discuss some issues associated with the ab-initio treatment of ionised impurity scattering, where the potential of the impurities is included directly in the dynamics of a Monte Carlo simulation rather than being introduced as a scattering rate. We have studied several methods for the solution of Poisson's equation and the integration of the equation of motion to evaluate

\footnotetext{
* Corresponding author.
} 
their efficiency and accuracy. For Poisson's equation we compared a mesh-based solution with a near-exact potential based on Ewald summation [4]. The equation of motion was integrated either with a high-order adaptive Runge-Kutta scheme, for free carriers and impurities alone, or with a low-order Euler method within Brownian dynamics [5], which also includes phonons and proves to be more forgiving.

These methods were tested by calculating the mobility of electrons in a slab with randomly distributed impurities. Their application is also illustrated with the simulation of a $0.08 \mu \mathrm{m}$ channel length dual gate GaAs MESFET.

\section{COMPUTATIONAL ASPECTS}

The simplest approach to include individual impurity charges in a particle-based device simulation is to ascribe them to a mesh and to solve Poisson's equation as a boundary value problem. This method is efficient and works for devices with arbitrary boundaries. Unfortunately, difficulties arise close to charges where the $1 / r$ Coulomb potential diverges. The errors in calculating the interparticle forces from a mesh-based solution of Poisson's equation along the major axis of symmetry of a cubic mesh cell are illustated in Figure 1. The errors are significant when the distance between the charges becomes smaller than 2-3 mesh spacings. It is practical to correct the short-range part of the interaction estimated from the mesh analytically. For a 3D solution of Poisson's equation on an uniform mesh we use a simple linear interpolation for the corrections which reflects the symmetry of the mesh cell.

Direct analytic evaluation of the Coulomb forces between point charges can give accurate results but becomes to costly when large numbers of particles are involved in the simulation, or where the boundaries of the device are complex. For simple cases, with periodic boundary conditions, the method of Ewald summation [4] can be used. We have used a polynomial approximation

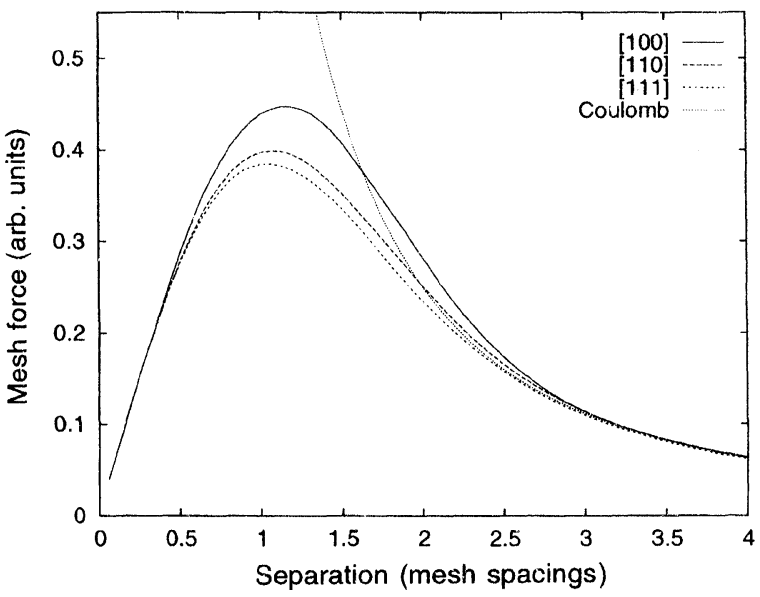

FIGURE 1 The forces between two point charges on along the main axes of symmetry of a cubic mesh cell. The true Coulomb force has been shown for comparison.

to the Ewald sums with fitted coefficients and compared the results with mesh-based solution of Poisson's equation.

Integration of the equations of motion in the complex potential landscape associated with the individual charges poses problems because the magnitude of the Coulomb force varies rapidly near the impurities. This necessitates the use of high-order integration schemes and small timesteps for free carriers. In this case we use an adaptive Runge-Kutta approach. Errors accumulate during the simulation and tight tolerances are therefore required.

Phonon scattering can be included using Brownian dynamics [5]. This is essentially an ensemble Monte Carlo method with a greatly simplified scattering term. Low-order Euler integration of the equation of motion is satisfactory in this case because the interaction with the phonons built into Brownian dynamics tends to erase errors in the integration of forces.

\section{IONISED IMPURITY SCATTERING}

The methods discussed above have been tested by calculating the mobility of electrons in bulk GaAs at room temperature. The crystal was represented 
as a periodic array of cubic cells and ionised impurity scattering was introduced through the potential of randomly distributed impurities in each cell. A uniform background charge was added to maintain overall neutrality. Poisson's equation was solved using a multigrid technique for the mesh-based methods. A typical pontential distribution in a $(50 \mathrm{~nm})^{3}$ cell is given in Figure 2. The same positions of impurities were used in the Ewald summation approach.

The mesh spacing must not exceed the distance at which the Coulomb interaction equals the thermal energy - about $2.8 \mathrm{~nm}$ at room temperature. In our calculations we used a $1 \mathrm{~nm}$ mesh. It is natural to assume that the cell size has to be larger than the mean free path of the carriers. For doping concentration $N_{D}=10^{24} \mathrm{~m}^{-3}$ the mean free path is approximately $50 \mathrm{~nm}$ and a mesh with more than $50^{3}$ points is therefore needed. This introduces a problem for lower concentrations where the mesh has to be even larger. However, experiments for $N_{D}=10^{24} \mathrm{~m}^{-3}$ with meshes ranging from $10^{3}$ to $200^{3}$ points gave a very small spread in the calculated mobility, $\mu_{n}=0.417 \pm 0.008 \mathrm{~m}^{2} / \mathrm{Vs}$. The history of 65000 particles was followed in the simulation and the diffusion coefficient was calculated from the variance in the range using a

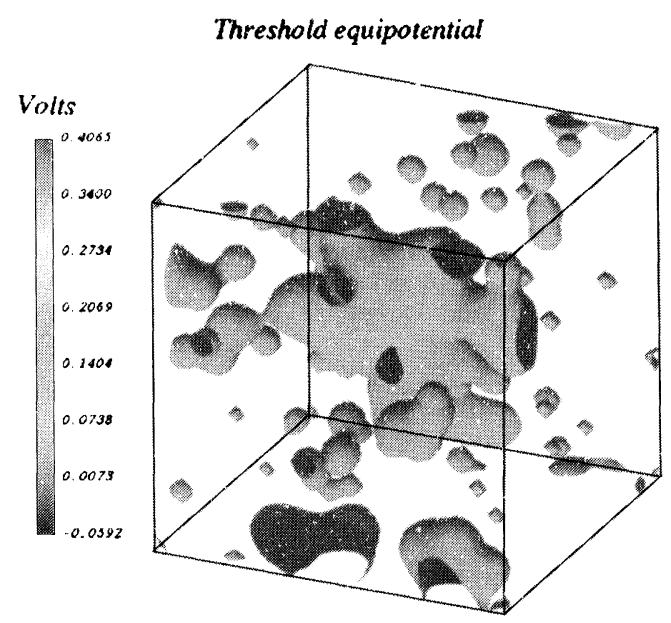

FIGURE 2 Random potential in a uniformly doped slab near the percolation threshold. least-squares fit. The mobility was then deduced from the Einstein relation.

The results are summarised in Figure 3 and compared with the most commonly used analytical formulas. Phonon scattering was excluded from the Brownian simulations using Mattheisen's rule where necessary. The Brownian dynamics simulation with a mesh-based solution of Poisson's equation is in good agreement with the ConwellWeisskopf formula over the whole range of doping. Brownian dynamics in combination with the more accurate Ewald sum for Poisson's equation gives lower mobility than the mesh-based results. The results for the Ewald summation and free carrier dynamics show a marked reduction in mobility which is more pronounced as the doping level is increased. The most likely cause of this phenomenon is the trapping of carriers in the potential well at the site of each impurity. The lack of phonon scattering means that is no mechanism for the electrons to exchange energy with the crystal and reach thermal equilibrium. It is therefore important to impose the correct initial distribution of carriers. The use of an unmodified Maxwell-Boltzmann distribution to initiate the simulation would pose severe problems because it predicts that all carriers are trapped in the Coulomb wells.

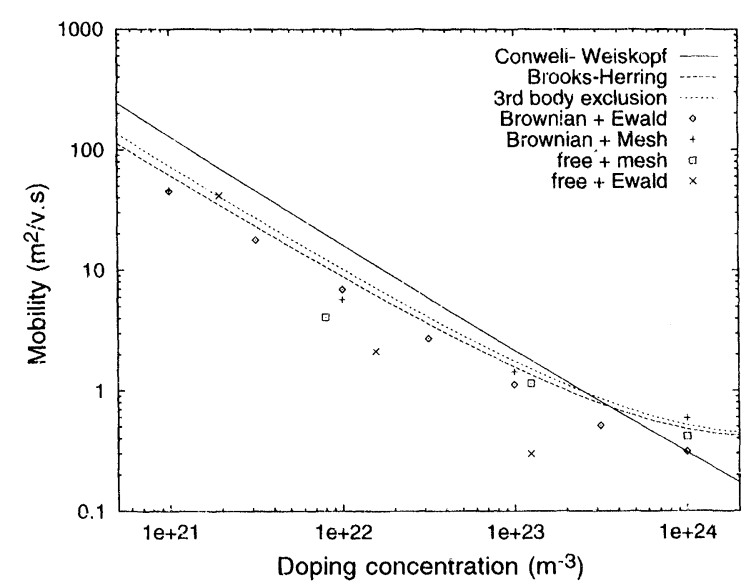

FIGURE 3 Mobility as a function of the doping concentration. 


\section{DUAL GATE MESFET EXAMPLE}

The Brownian method on a mesh was used in a simple simulation of an $80 \mathrm{~nm}$ dual-gate MESFET with $N_{D}=5 \times 10^{23} \mathrm{~m}^{-3}$ in the channel. Figure 4 illustrates the potential in the channel near threshold; fluctuations due to the random donors are evident. Figure 5 shows that the results of this atomistic simulation agree well with drift-diffusion results from MEDICI, at low source-drain voltage. The slope of the $I_{D}\left(V_{G}\right)$ characteristic is proportional to the mobility, and shows that impurity scattering is properly included in the Brownian simulation.

\section{CONCLUSIONS}

We have considered different approaches to the solution of both Poisson's equation and the equation of motion in a simulation where ionized impurities are treated as discrete random charges. We conclude that the simpler mesh-based solution of Poisson's equation does not introduce signifi-

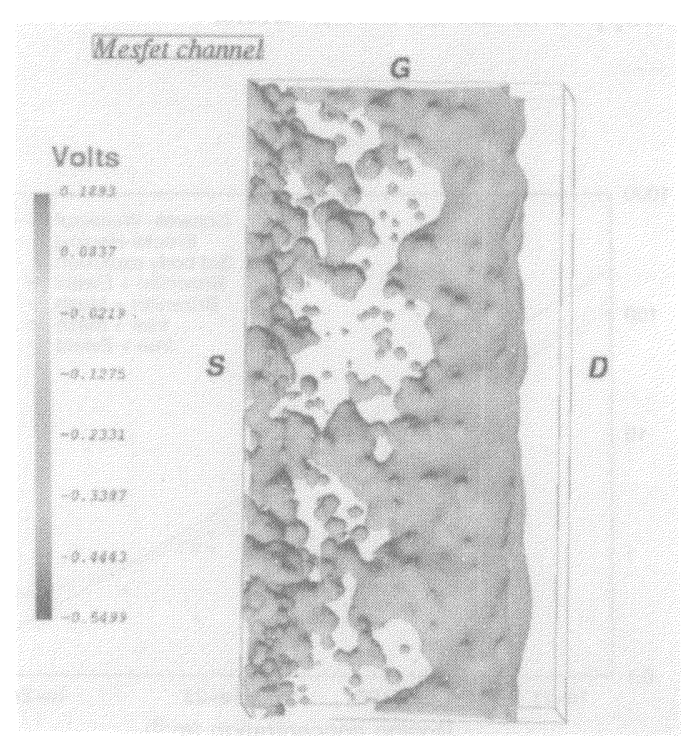

FIGURE 4 Equipotential surfaces in the channel of $80 \mathrm{~nm}$ channel dual gate GaAs MESFET at threshold.

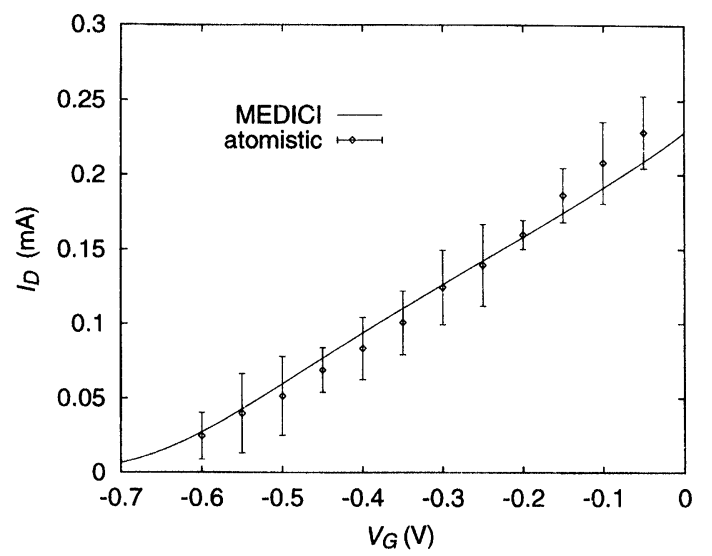

FIGURE 5 Transfer characteristics of an $80 \mathrm{~nm}$ channel dual gate GaAs MESFET, comparing the "atomistic" Brownian simulations and drift-diffusion MEDICI simulations at low drain voltage $\left(V_{D}=0.05 \mathrm{~V}\right)$.

cant errors, and reproduces well the expected mobility as a function of doping. Highly accurate integration of the equation of motion is needed for free carriers, but the interaction with phonons in the Brownian method makes it more tolerant of errors.

\section{References}

[1] Wong, H.-S. and Taur, Y. (1993). "Three-dimensional 'atomistic' simulation of discrete random dopant distribution effects in sub-0.1 $\mu \mathrm{m}$ MOSFETs", IEDM Tech. Digest, 705-708.

[2] Ferry, D. K., Kriman, A. M., Kann, M. J. and Joshi, R. P. (1991). "Molecular dynamics extension of Monte Carlo simulation in semiconductor device modeling", Computer Physics Communication, 67, 119-134.

[3] Tarnay, K., Masszi, F., Poppe, A., Verhas, P., Kocsis, T. and Kohari, Z. (1993). "A 3D Monte Carlo semiconductor device simulator for submicron MOS transistors", Software Applications in Engineering, CH-46, 345-358.

[4] Adams, D. J. and Dobey, G. (1987). J. Comp. Physics, $72,156$.

[5] Arokianathan, C. R., Asenov, A. and Davies, J. H. (1996). "An approach based on Brownian motion for the simulation of ultrasmall semiconductor devices", J. Applied Physics, 80(1), 1-7.

\section{Authors' Biographies}

Clinton Arokianathan graduated from Glasgow University with a B.Eng. in Physics and Electronic 
Engineering in 1993. He then undertook a Ph.D. at the Department of Electronics and Electrical Engineering, where he developed parallel and serial code for the molecular dynamics simulation of submicron devices. He now works on recognition systems and vision processing for Roke Manor Research in Romsey.

John Davies gained his Ph.D. from Cambridge University on aspects of electronic stages in amorphous semiconductors. He later worked on the quantum theory of high-field transport in semiconductors at Cornell. Since coming to Glasgow in 1986, much of his research has been concerned with the modelling of ultra-small structures used in quantum transport. In particular, long-ranged fluctuations from ionized donors were shown to have a serious effect on transport in wires and quantum point contacts. Other interests include resonant tunnelling and effects of stress in nanostructures.

Asen Asenov had 10 years industrial experience as a head of the Process and Device Modelling Group in IME-Sofia, developing one of the first integrated process and device CMOS simulators IMPEDANCE. He is currently a Reader in the Department of Electronics and Electrical Engineering, Glasgow University. As a leader of the Device Modelling Group he is involved in the development of 2D and 3D device simulators and their application in the design of compownd FETs, SiGe MOSFETs and IGBTs. Other interests include design of parallel algorithms. 

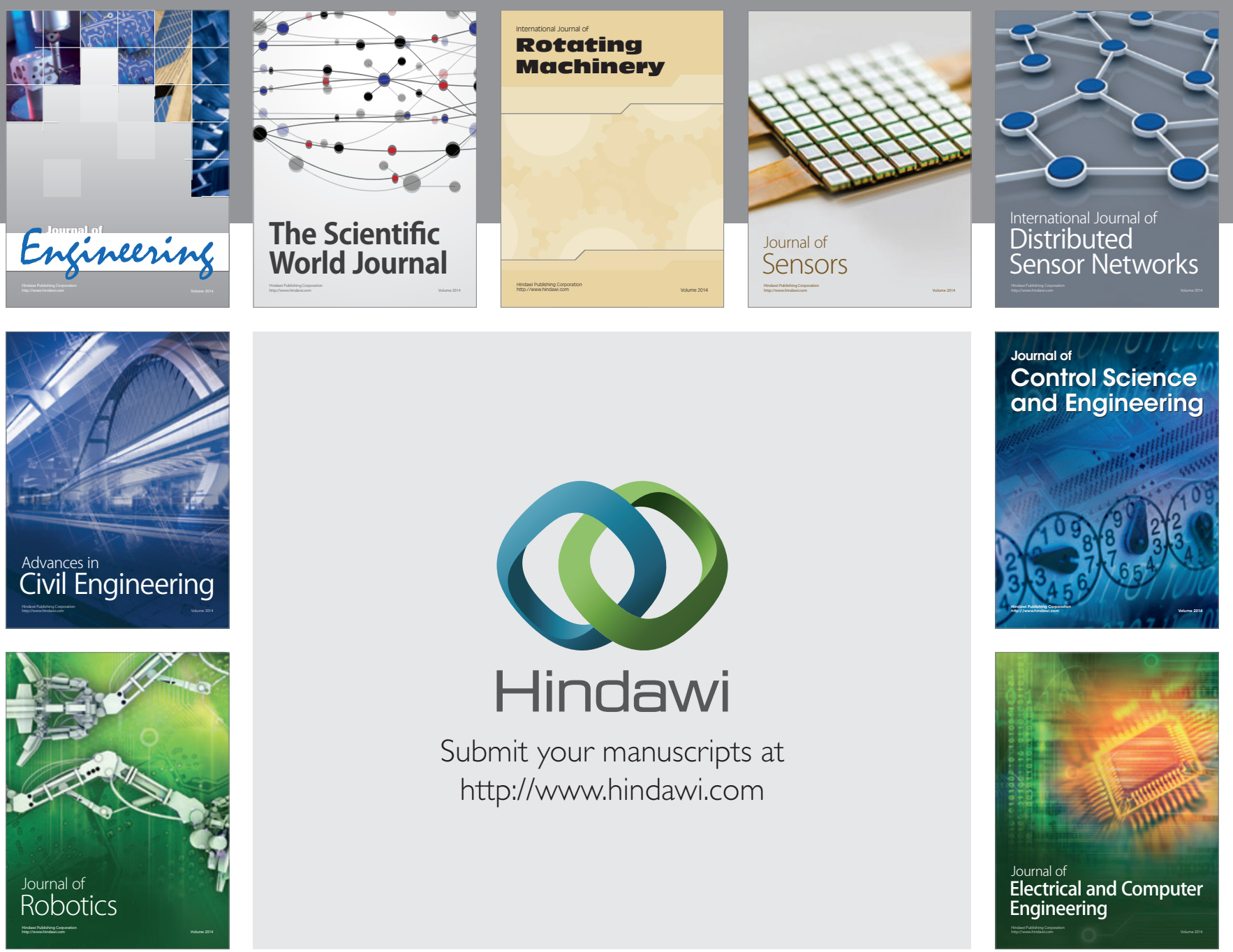

Submit your manuscripts at

http://www.hindawi.com
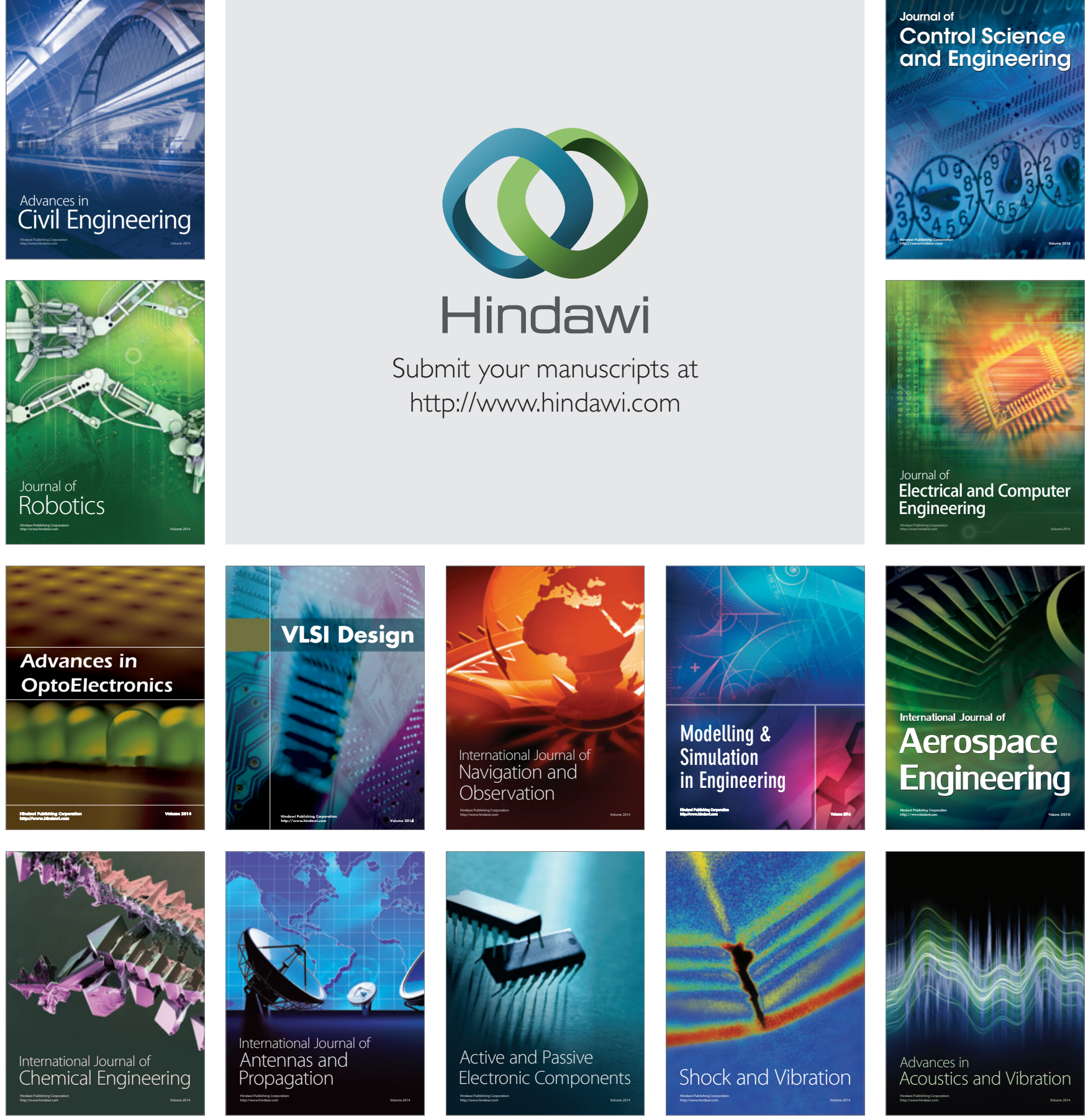